\title{
Pseudoporyphyria Associated with Lemon Water and Naproxen
}

\author{
Nwanneka Okwundu ${ }^{1 *}$, John Moesch ${ }^{2}$, Sarah Belden ${ }^{3}$ \\ ${ }^{1}$ Department of Dermatology, Huntsman Cancer Center, University of Utah, Salt-lake City, Utah \\ ${ }^{2}$ Department of Dermatology, Largo Medical Center, Largo, Florida \\ ${ }^{3}$ Department of Dermatology, Case Western Reserve University, Cleveland, Ohio
}

Received: March 05, 2020; Accepted: May 05, 2020; Published: June 18, 2020

*Corresponding author: Nwanneka Okwundu,University of Utah Department of Dermatology, Salt-lake City, Utah, USA. E-mail: Nwanneka. okwundu@hci.utah.edu

\begin{abstract}
Background: Pseudoporyphyria is an uncommon bullous dermatosis It shares clinical and histological features with porphyria cutanea tarda, but it occurs in the absence of porphyrin metabolic dysfunction. It is characterized by skin fragility, bullae, milia, and scarring on the dorsum of the hands and other sun-exposed areas.

Case: We present a patient on naproxen with recurrent pseudoporyphyria of the dorsal hands associated with the consumption of lemon water.

Findings: Biopsy showed a pauci-inflammatory sub epidermal vesicle with caterpillar bodies and laboratory studies lacked any urine or serum porphyrin abnormalities. The cutaneous lesions were noted to resolve with discontinuation of the consumption of lemon water.

Keywords: Pseudoporyphyria; Porphyrin; Porphyria Cutanea Tarda; Chlorophyll; Vesicle and Depigmented
\end{abstract}

\section{Introduction}

Pseudoporyphyria is a rare photodermatosis with clinical and histological features similar to those of porphyria cutanea tarda, but lacks the abnormalities seen in porphyrin metabolism [1]. This skin photosensitivity disorder has been associated with some known etiological agents. The two most common causes include ultraviolet (UV) radiation and medications [2]. Patients may develop bullae and vesicles on sun exposed areas such as the dorsum of the hands, forearms, face, lower legs, and feet [3]. They may also present with skin fragility and easy bruising after minor trauma. Bullae heal with scarring and milia in most patients. Pseudoporyphyria is often difficult to treat and except for photoprotection and suspension of possible medications involved, there is currently no specific treatment regimen [4].

\section{Case report}

A 60-year-old Caucasian male presented with intermittently recurrent vesicles on the dorsal hands for the past 15 years. Patient denied any family history of blisters. He worked as a landscaper for a golf course. His past medical history included arthritis and vitiligo. Medications included alendronate, cyclobenzaprine, fluticasone, tramadol and naproxen, celecoxib. Of note, patient reported being on the medication regimen for several years before the initial blister outbreak. Patient reported that biopsies of the lesions were performed in the past with inconclusive results. Patient stated that the blisters appeared shortly after he began ingesting lemon water; he would cut up lemons and leave them in his water overnight to consume the following day. Patient reported that whenever he did not consume the lemon water, the blisters would resolve.Dermatologic examination revealed tense bullae and vesicles isolated to the dorsal hands. Also, on the dorsal hands were scattered depigmented patches consistent with the patients known history of vitiligo(Figures 1,2). Patient was instructed to discontinue naproxen and to follow up in 6 weeks. At follow-up, tense vesicles were present. Labs were drawn and were within normal limits, such as hepatitis panel, complete blood count with differential, complete metabolic profile (including the blood urea nitrogen, creatinine, sodium, iron, and magnesium, and calcium levels). A 24-hour urine quantitative porphyrin analysis and serum porphyrin were normal.

Punch biopsy showed a pauci-inflammatory sub epidermal vesicle with caterpillar bodies (Figure 3). Skin immunofluorescence showed focal positivity for IgG in superficial vessel walls and skin. PAS stain was negative for fungal organisms. Differential diagnosis for pseudoporyphyria include erythropoietic protoporphyria, variegate porphyria, hereditary coproporphyria, congenital erythropoietic porphyria, and other bullous disorders presenting with similar clinical manifestations, including epidermolysis bullosa acquisita, bullous pemphigoid, bullous lupus erythematosus, and hydroa vacciniforme. However, all of these are histopathologically and biochemically different from pseudoporyphyria and the unremarkable 24-hour urine quantitative porphyrin analysis, serum porphyrin, and other laboratory results make them unlikely as well. 

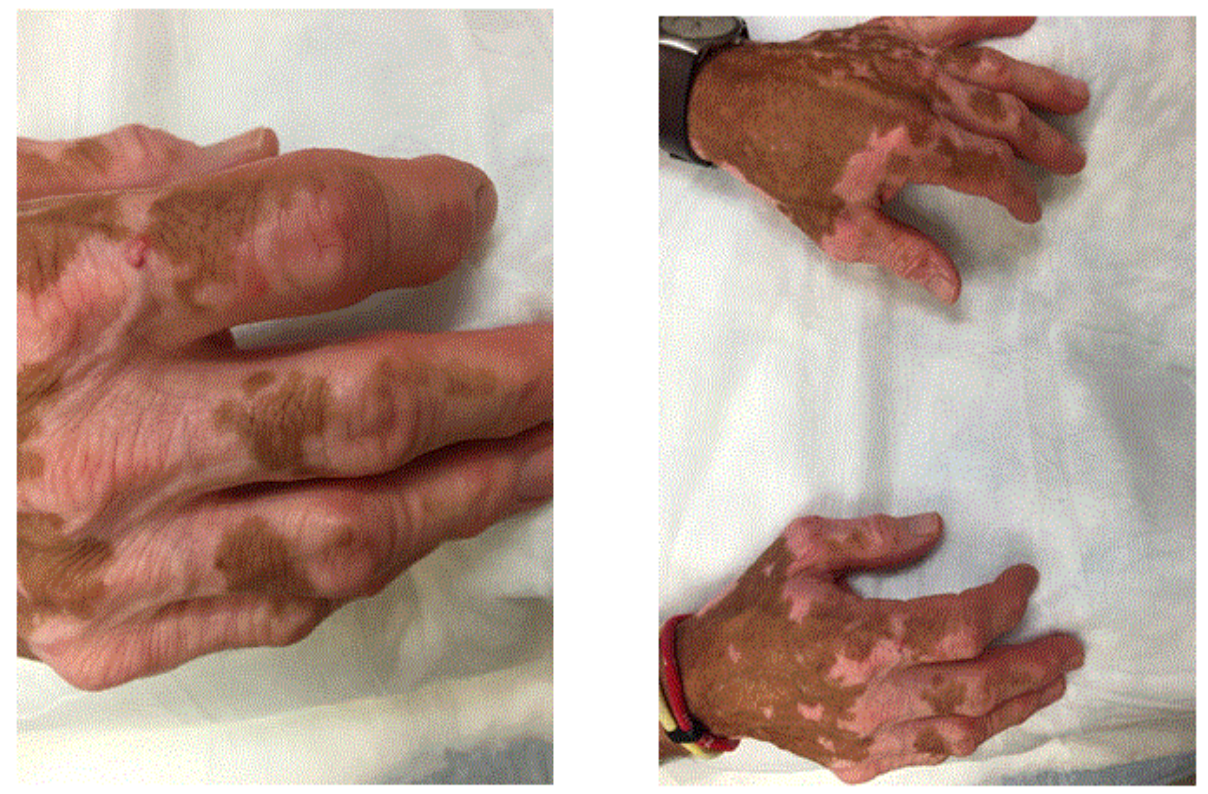

Figure 1 \& 2: Depigmented Patches on Dorsal Hands

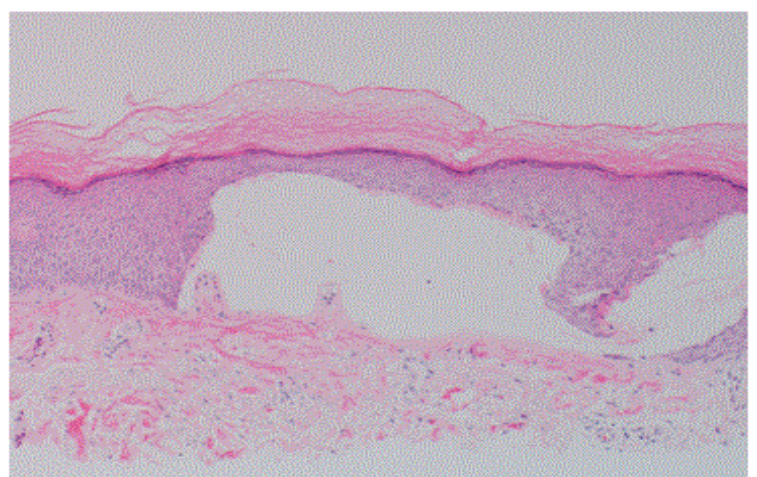

Figure 3: Pauci-inflammatory sub epidermal vesicle with caterpillar bodies

\section{Discussion}

Pseudoporyphyria (PP) is a rare bullous dermatologic disorder that commonly occurs after exposure to phototoxic or photosensitizing chemicals. Cutaneous and histologic manifestations of pseudoporyphyria are the same as poryphyria cutanea tarda (PCT) with the appearance of skin fragility features. Clinical manifestation of these conditions include bullae, vesicles, and scarring on superficial sun exposed skin, and histology characteristically shows non-inflammatory, subepidermal bulla with pauci perivascular lymphocytic infiltrate [5]. However, pseudoporphyria can be differentiated from poryphyria cutanea tarda by the presence of normal porphyrin levels in plasma, erythrocytes, urine, and stool [6]. Furthermore, porphyria cutanea tarda may have associated symptoms such as hypertrichosis, hyperpigmentation, and sclerodermoid changes, all of which are rarely seen in pseudoporyphyria [7].

The thickness of the blood vessel wall could also be helpful in differentiating PP from PCT [8]. A comparative histological study was done on biopsy samples of patients with PCT and PP. It was found that the blood vessel walls were thickened in 11 of 13 patients with PCT while only 1 out of 9 of the blood vessel walls in PP patients displayed these features. Other notable features of PP are the presence of IgG, IgM, C3, and fibrinogen on direct immunofloresence on the vessel walls and at the dermalepidermal junction of PP patients [9].

Diagnosis of PP requires an underlying trigger. The most common reported triggers include the potentially phototoxic medications such as naproxen, nabumetone, furosemide, ciprofloxacin, acitretin, voriconazole [10,11]. However, pseudoporphyria manifests with vesicles and bullae, whereas drug-induced phototoxic reactions usually appear as exaggerated sunburns. The reason for this is unknown. Other reported associations include history of chronic renal failure and dialysis. The suspected photosensitizing agent in dialysis solution is aluminum hydroxide or chemicals in polyvinyl chloride dialysis tubing $[12,13]$.

PP has also been reported with exposure to chlorophyll in various forms [14]. Chlorophyll is a natural constituent of the human diet, occurring naturally in green leafy vegetables such as spinach, and is usually metabolized without causing any symptoms. However, some chlorophyll metabolites are known to be active photosensitizers [15]. Chlorophyll-derived 
photosensitization has been found in ruminant livestock and photosensitizing metabolites such as pheophorbide-a and phytoporphyrin. It has also have been demonstrated in Japanese patients who were self-medicating with commercial extracts of Chlorella algae [14].

Furthermore, in previous studies, researchers have found a substance called pheophorbide-a that results from the chemical procedures used to extract the chlorophyll from the plant. Chlorophyllase is an enzyme present in plants and is resistant to temperatures lower than $80^{\circ} \mathrm{C}$. Lower temperatures result in chlorophyllide formation followed by non-enzymatic conversion in acidic conditions to pheophorbide-a. In Australia, chlorophyll is marketed by several vitamin companies as a natural super antioxidant food supplement. This chlorophyll has been reported as a known trigger for pseudoporyphyria $[14,16,17]$. The lemon water which the patient ingested may contain pheophorbide-a which may be responsible for the outbreaks

Another possible trigger for our patient's PP is the use of naproxen for pain relief [18]. Naproxen has been reported as a known precipitant of pseudoporyphyria, especially in children. Although this is a possible cause for some of his outbreaks, in the history elicited from the patient, he denied the eruption of new cutaneous lesions with naproxen

Treatment of pseudoporphyria includes stopping the offending agent. Prognosis can be very good if the causative agent is identified and discontinued. The resolution of the clinical manifestations is slow with complete resolution in a period of months to years. Sometimes a secondary infection may even occur [19]. There are certain measures that may help in the management of pseudoporyphyria. Photoprotection such as use of sunscreens, sun avoidance, and use of protective clothing is essential for all patients with pseudoporphyria. Photo-protection should continue until resolution of symptoms. If pseudoporphyria is associated with excessive exposure to artificial UVA sources from tanning beds, the patient should be cautioned to discontinue this practice. $\mathrm{N}$-acetylcysteine has been reported to have a beneficial effect in a few patients with chronic renal failure and hemodialysis-associated pseudoporphyria. $\mathrm{N}$-acetylcysteine is a precursor of glutathione, an endogenous antioxidant, the levels of which are reduced in hemodialysis patients. Hemodialysis patients have also been reported to respond to oral glutamine, photoprotection, and vitamin D supplementation [20,21].

\section{Conclusion}

Chlorophyll ingestion from consumption of lemon water may have been a key precipitating factor for this patient's recurrent pseudoporyphyria. However, there is a possibility that his outbreak may have been multifactorial given his chronic use of NSAID agents and ultraviolet light exposure from his outdoor job. In his case, treatment would entail eliminating all possible triggers. We present this case to raise awareness of this uncommon dermatologic diagnosis which may have multiple underlying factors contributing to its development, but specifically attributed to lemon water based on history.

\section{References}

1.Green JJ, Manders SM. Pseudoporphyria. J Am Acad Dermatol. 2001;44(1):100-108.

2. Schad SG, Kraus A, Haubitz I, Trcka J, Hamm H, Girschick HJ. Early onset pauciarticular arthritis is the major risk factor for naproxeninduced pseudoporphyria in juvenile idiopathic arthritis. Arthritis Res Ther. 2007;9(1):R10.

3. Czop BB, Wcisło BL. Pseudoporphyria induced by hemodialysis. Advances in Dermatology and Allergology. 2014;31(1): 53-55.

4. Pavanelli GM, MilanoSS, Sevignani G, Jung JE, Funke VAM, Nascimento MMD. Furosemide-induced pseudoporphyria in a patient with chronic kidney disease: case report. Brazilian Journal of Nephrology. 2018;40(3):287-290.

5. Weidner T, Tittelbach J, Schliemann S, Goetze S, Elsner P. Blisters, ulcers, crusts, and atrophic scars on the back of the hands and the extensor aspects of the forearms. J Dtsch Dermatol Ges. 2018;16(1):88-91.

6. Kochs C, Mühlenstädt E, Neumann NJ, Hanneken S. Solarium-induced pseudoporphyria and variegate porphyria as rare differential diagnoses of porphyria cutanea tarda. Hautarzt. 2009;60(10):790793.

7. Beer K, Applebaum D, Nousari C. Pseudoporphyria: discussion of etiologic agents. J Drugs Dermatol. 2014;13(8):990-992.

8. Quaiser S, Khan, R, Khan AS. Drug induced pseudoporphyria in CKD: A case report. Indian Journal of Nephrology. 2015;25(5):307-309.

9. Maynard B, Peters MS. Histologic and immunofluorescence study of cutaneous porphyrias. Journal of Cutaneous Pathology. 1992;19(1):40-47.

10.Tolland JP, Mckeown PP, Corbett JR. Voriconazole-induced pseudoporphyria. Photodermatology, Photoimmunology \& Photomedicine. 2007;23(1):29-31.

11.Schmutz JL, Barbaud A, Tréchot P. Ciprofloxacin and pseudoporphyria. Ann Dermatol Venereol. 2008;135(11):804.

12. Masmoudi A, Ben Hmida M, Mseddi M, et al. Cutaneous manifestations of chronic hemodialysis. Prospective study of 363 cases. Presse Med. 2006;35(3 Pt 1):399-406.

13. Massone C, Rudolph CM, Stefani A, Müllegger RR. Successful outcome of haemodialysis-induced pseudoporphyria after short-term oral $\mathrm{N}$-acetylcysteine and switch to high-flux technique dialysis. Acta Derm Venereol. 2006;86(6):538-540.

14. Rossi E, Borchard K, Cole JM. Pseudoporphyria following selfmedication with chlorophyll. Australasian Journal of Dermatology. 2014;56(1):47-48.

15. Zhao CY, Frew JW, Muhaidat J, Cheung K, Lee P, Poulos $\mathrm{V}$, et al. Chlorophyll-induced pseudoporphyria with ongoing photosensitivity after cessation - a case series of four patients. Journal of the European Academy of Dermatology and Venereology. 2016;30(7):1239-1242. 
16. SaadHS, Azoulay T, Arazi T, Yaakov BE, Mett A, Shiboleth YM, et al. Chlorophyllase Is a Rate-Limiting Enzyme in Chlorophyll Catabolism and Is Posttranslationally Regulated. The Plant Cell. 2007;19(3):1007-1022.

17. Tang PMK, Xuan NH, Wong CK, Fong WP, Fung KP. Pheophorbide a-Mediated Photodynamic Therapy Triggers HLA Class I-Restricted Antigen Presentation in Human Hepatocellular Carcinoma. Translational Oncology. 2010; 3(2):114-122.

18. Burns DA. Naproxen pseudoporphyria in a patient with vitiligo. Clin Exp Dermatol. 1987;12:296-297.
19. Guiotoku MM, Pereira Fde P, Miot HA, Marques ME. Pseudoporphyria induced by dialysis treated with oral $\mathrm{N}$-acetylcysteine. An Bras Dermatol. 2011;86(2):383-385.

20. Seyedi VJ, Dobbeleer G, Simonart T. Treatment of hemodialysisassociated pseudoporphyria with N-acetylcysteine: Report of two cases. Br J Dermatol. 2000;142(3):580-581.

21. Garg T, Barara M, Chander R, Meena S, Agarwal S. Pseudoporphyria: A Case Report. Indian Journal of Dermatology. 2015;60(3):324. 